\title{
Toxicity of Visible Light Follows a Rule Similar to that for X-Ray Damage
}

\section{Rino Saiga and Ryuta Mizutani*}

Department of Applied Biochemistry, Tokai University, Hiratsuka, Kanagawa 259-1292, Japan

*ryuta@tokai-u.jp

The paper entitled "Less is More: Longer Exposure Times with Low Light Intensity is Less Photo-Toxic" published in the November 2017 issue of Microscopy Today [1] reports an interesting study regarding the relationships between the light intensity and its biological effects. The authors of this paper suggested that one can eliminate the photo-toxicity by lowering "temporal light dose." We would like to propose an alternative parameter that can better describe the results: light power.

In the abovementioned paper, the "temporal light dose" $(\mathrm{mW} / \mathrm{s})$ seems to be calculated by dividing the light power $(\mathrm{mW})$ by the exposure time (s). However, the light power $(\mathrm{mW})$ itself is defined as the rate of energy transfer per second $(\mathrm{mJ} / \mathrm{s})$ and is already divided by time. The dimension of the "temporal light dose" represents time variation of the light power, although no information regarding the time variation of the light power is presented in the paper.

A plausible parameter "light dose" $(\mathrm{mJ})$ can be calculated by multiplying the light power $(\mathrm{mW})$ by the exposure time (s). For the conditions shown in Table 1 of the abovementioned paper, the light dose is $0.57,0.47,0.42$, or $0.39 \mathrm{~mJ}$ for the experiment using the light power of 1.64, 6.68, 12.03, or $22.10 \mathrm{~mW}$, respectively. Differences between these light doses are small. This is already stated by the authors that "the total light exposure to the sample was similar for each image". Therefore, the light power rather than the light dose should be the essential factor of the photo-toxicity.

Figure 1 of the abovementioned paper [1] shows a pseudo-linear relationship between the photo-bleaching decay rate and the "temporal light dose." However, the decay rate shows an exponential correlation by plotting it against the light power (Figure 1 (revised)).

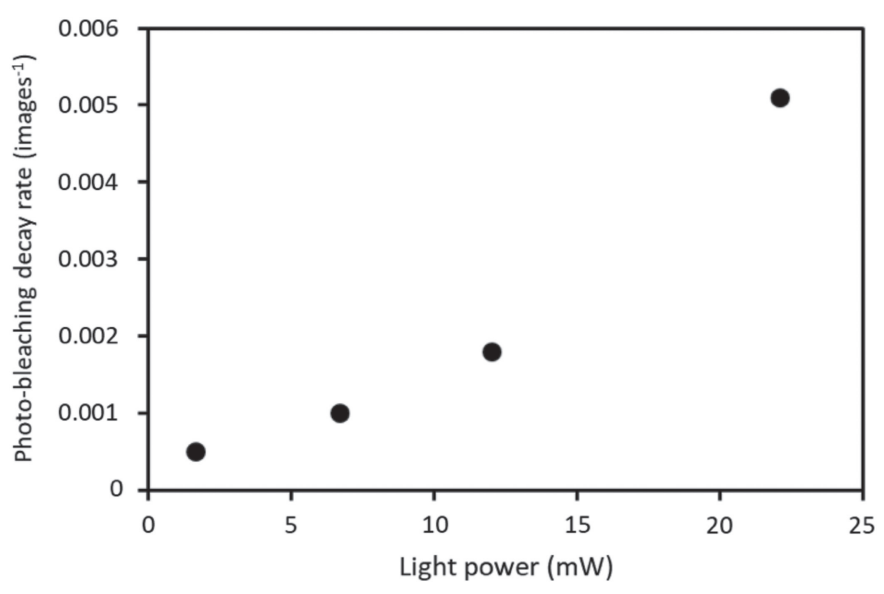

Figure 1 (revised): Photo-bleaching rates for fixed cells.

This is consistent with the exponential model of the biological effect of X-ray or UV irradiation [2], suggesting that the toxicity of visible light follows a rule similar to that of the $\mathrm{X}$-ray damage. Thus, Figures 2, 3, 7, and 8, as well as the text and Table 1 [1], should be reinterpreted by taking this into account. The results indicate that the photo-toxicity can be eliminated in an exponential manner by lowering the light power. This reinterpretation will not impair the paper contents, but rather it strengthens the findings originally reported in the abovementioned paper [1].

\section{References}

[1] F Mubaid and CM Brown, Microscopy Today 25(6) (2017) 26-33.

[2] L Bodgi et al., J Theor Biol 394 (2016) 93-101.

\section{Authors' Response}

\section{Firas Mubaid and Claire M. Brown*}

Department of Physiology, McGill University, Montreal, Canada, H3G 0B1

*claire.brown@mcgill.ca

In their Letter to the Editor statement above, Saiga and Mizutani make some insightful comments regarding the parameters used in our paper "Less is More: Longer Exposure Times with
Low Light Intensity is Less Photo-Toxic." We thank the authors for taking the time and bringing these issues to our attention. The authors are correct that the major factor affecting photo-bleaching 